\title{
Genetic Linear Averaging Algorithm for Zooming Digital Images
}

\author{
Baydaa Sulaiman Bahnam \\ baydaa_sulaiman@uomosul.edu.iq
}

College of Computer Science and Mathematics

University of Mosul, Mosul, Iraq

\section{Received on: 12/12/2011}

\section{ABSTRACT}

Accepted on: 19/04/2012

In this paper, the hypernation of linear averaging algorithm for zooming images is achieved with genetic algorithm. It's applied on a number of samples of images that lack the indistinction of the outline and providing accurate images. The equation of linear averaging is utilized as an objective function in genetic algorithm using several types of crossovers and mutations. A compression among these types is accomplished using two measures (RMSE \& PSNR) for evaluating the proposed algorithm. The ratio for zooming is twice as the original images. The accuracy and the efficiency of those images are RMSE $=6.6541$ and PSNR $=31.6470 \mathrm{db}$. A MATLAB 7.10.0(R2010a) environment is used for the programming of proposed algorithm will all applied types of crossovers and mutations.

Keywords: Genetic Algorithm, Linear Averaging Algorithm, Zooming Images, Digital Images.

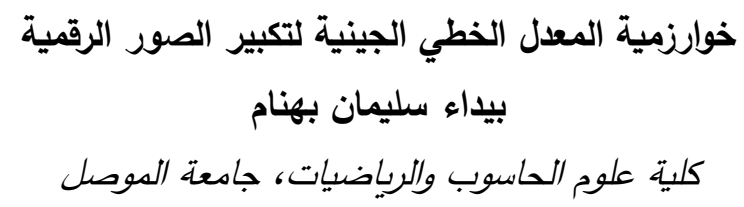

تاريخ قبول البحث: 2012/04/19

تاريخ استلام البحث: 2011/12/12

\section{الملخص}

تم في هذا البحث تهجين خوارزمية المعدل الخطي لتكبير الصور مع الخوارزمية الجينية وتطبيقها على

عدد من النماذج الصورية التي تعاني من عدم الوضوح في المعالم وتهيئة الصور بشكل أفضل وأدق. وتم اعتماد معادلة المعدل الخطي بوصفها دالة هدف في الخوارزمية الجينية واستخدام عدة أنواع من التداخلات والطفرات وإجراء مقارنة بين هذه الأنواع واستخدام مقياسي (PSNR \&RMSE) لتقييم كفاءة الخوارزمية المقترحة وكانت

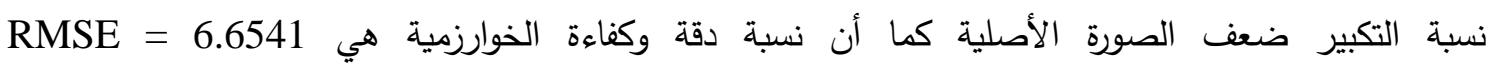
و لصورة PSNR = 31.6470 db. وتم برمجة الخوارزمية المقترحة مع جميع أنواع التداخلات

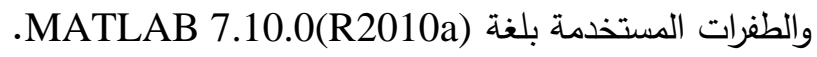
الكلمات المفتاحية: الخوارزمية الجينية، خوارزمية المعدل الخطي، تكبير الصور، الصور الرقمية. 1. المقدمة

تعد عملية تكبير الصورة Zooming من أكثر العمليات المستخدمة على الصورة ، إذ تكاد لا تخلو أي

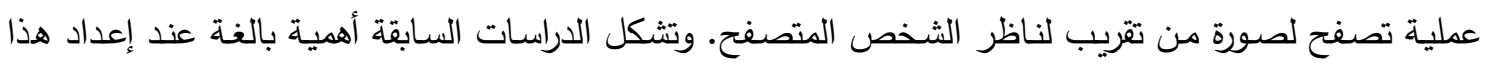
البحث ومن هذه الدراسات:

1. قدمت دراسة Raheema طريقة لتكبير الصور وذلك بنشر قيمة كل نقطة ضوئية في الصورة الداخلة إلى كل النقاط الضوئية المجاورة له بمربع 3*3 في مواقع الصورة الخارجة مع عمل ترشيح للصورة باستخدام طريقة 
2. وتتاولت دراسة Valizadeh وآخرين طريقة لتكبير الصور الملونة والرمادية بالاعتماد على Moving Least

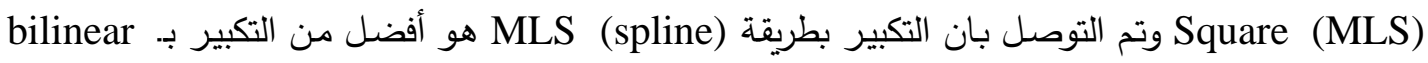
interpolation 3. في حين قدمت دراسة Battiato وآخرون تقنية جديدة لتكبير الصور الرقمية في نطاق التدرج الرمادي والملون

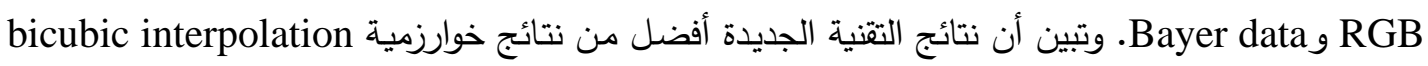
.[3] pixel replication, 4. أما دراسة Romero Almira واقترحت طريقتين لتكبير الصور الرمادية اعتمدت على sampling theory

وفي هذا البحث تم استخدام إحدى الحلـول الذكائيـة إلا وهـي الخوارزميـة الجينيـة وتهجينها مـع إحدى الخوارزميات التقليدية في تكبير الصور وهي خوارزمية المعدل الخطي. وعليه تم بناء خوارزمية جينية دالة هدفها خوارزمية المعدل الخطي وتطبيقها على صور ذات تدرج رمادي. وتم تطبيق عدة أنواع من التداخلات والطفرات وإجراء المقارنة فيما بينهم لبيان الأفضل.

وسيتم عرض في المقطع 2 أهداف البحث ، ويتضمن المقطع 3 تعريف واستخدامات الخوارزمية الجينية ، في حين أن اغلب مجالات تكبير الصور سيتم عرضها في المقطع 4 فضلا عن خطوات خوارزمية المعدل الخطي، أمـا المقطع 5 فتتـاول خطـوات خوارزميـة المعدل الخطـي الجينيـة المقترحـة والتي تـم اختبارهـا في 6 ، وأخيـرا الاستتناجات والبحوث المستقبلية في المقطع 7 والمصادر في 8.

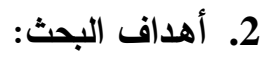

1. صياغة خوارزمية المعدل الخطي الجينية المقترحة لتكبير الصور الرقمية.

2. اختبار الخوارزمية المقترحة للتحقق من مدى ملائمة الخوارزمية المصممة لتكبير الصور الرمادية. 3. مقارنة بين خوارزمية المعدل الخطي الجينية المقترحة وخوارزمية المعدل الخطي التقليدية باستخدام مقياسين .PSNR g RMSE 4. تطبيق عدة أنواع من الطفرات mutation والتداخلات crossover على الخوارزمية المقترحة وإجراء المقارنـة فيما بينهم.

3. الخوارزمية الجينية (Genetic Algorithm)

تعرف الخوارزمية الجينية بأنها خوارزمية ذكية يمكن استخدامها لحل المسائل المعقدة. وتعد من طرائق البحث الكفوءة المعتمدة على مبادئ الاختيار الطبيعي وعلم الوراثة ، ابتكرها العالم جون هولاند عام 1975 في جامعة ميشيكان[9][10].

وقد استخدمت الخوارزمية الجينية بشكل واسع في العديد من المجالات منها: معالجة الصور ( Image)

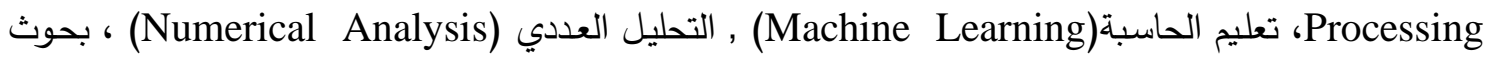
العمليات (Operations Research) ، وحل مسائل التشفير وكسر الشفرة (Cipher \& Decipher) وغيرها من المجالات التي لاقت بها نجاحا كبيرا [1] [15]. 
4. تكبير الصور (Image Zooming)

تعرف الصور الرقمية بأنها عينات منفصلة منظمة في فضاء معين وتضم بيانات رقمية تمثل كل قيمة منها

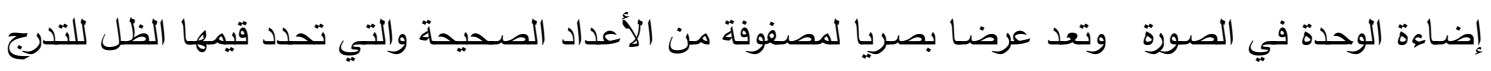
الرمادي في حالة الصور ذات التدرج الرمادي أو لون محدد في حالة الصور الملونة [2 ][4].

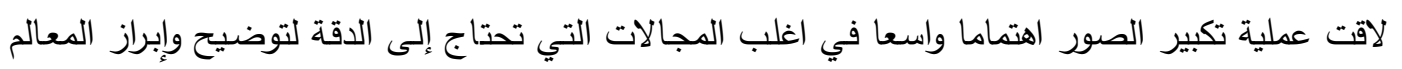

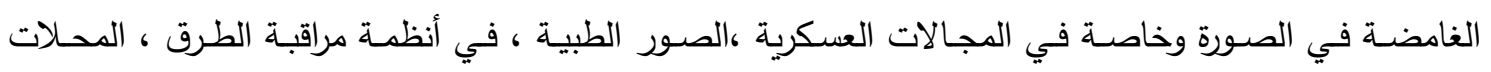

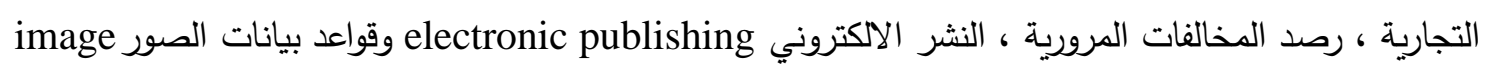
.[11] [6] [5] database ومن الطرائق المستخدمة في عملية تكبير الصور طريقة المعدل الخطي linear averaging التي تعدل

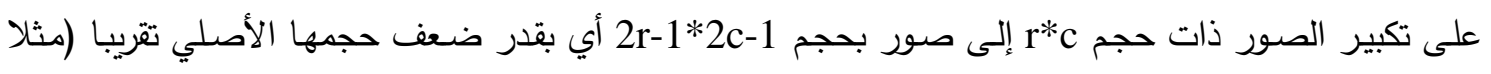
حجـم الصـور 128*128 سـيتم تكبيرهـا إلـى 255*255 ). وفيمـا يـي خطـوات خوارزميـة المعـدل الخطـي التقليدية[16][7] :

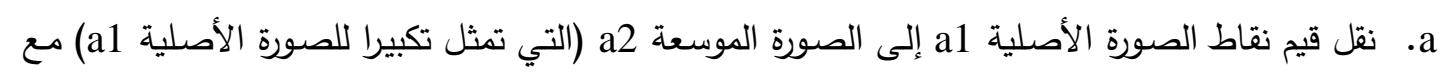
ترك نقطة فارغة بين كل نقطتين من نقاط الصورة الموسعة بالاتجاهين الأفقي والعمودي.

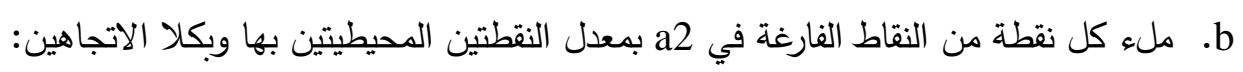

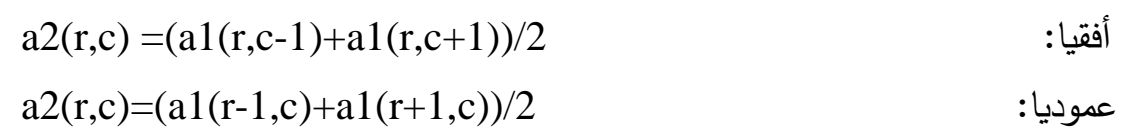
حيث ان r يمثل عدد الصفوف و c عدد الأعمدة في الصورة الأصلية.

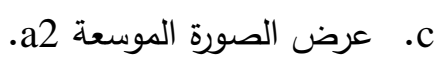

\section{5. خوارزمية المعدل الخطي الجينية المقترحة}

لقد تم استخدام خوارزمية المعدل الخطي بوصفها دالة هدف للخوارزمية الجينية المقترحة بهدف تكبير الصور • وان الخوارزمية المقترحة تعمل على تكبير الصورة بنسبة ضعف حجمها الأصلي تقريبا (أي تكبير صور ذات حجم r*c إلى صور بحجم 2r-1*2c-1 ) أو أكثر وحسب رغبة المستخدم ، كذلك بإمكانها تكبير جزء مقتطع من الصورة. وتم تطبيق عدة أنواع من التداخلات والطفرات في الخوارزمية المقترحة. أيضا تم برمجة هذه الته الخوارزمية المقترحة مع جميع أنواع التداخلات والطفرات المستخدمة بلغة (R2010a) وفيما يلي خطوات الخوارزمية المقترحة:

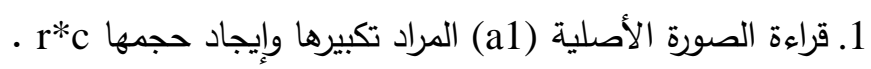

2. تهيئة وتحديد حجم الصورة الموسعة (a2) التي ستمثل تكبيراً بنسبة ضعف الصورة الصورة الأصلية تقريبا بحيث ان

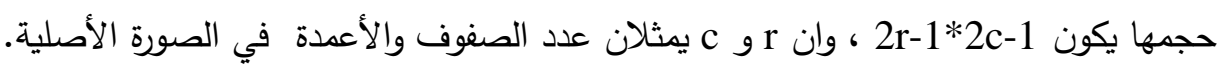

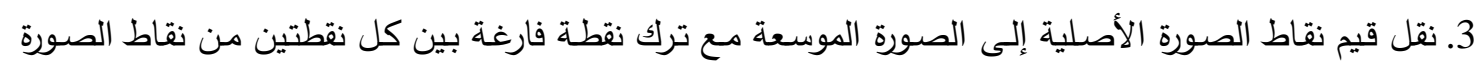
الموسعة بالاتجاهين الأفقي والعمودي.

4. تحديد المعاملات المستخدمة في الخوارزمية الجينية: يتم تحديد المعاملات المستخدمة في الخوارزمية الجينية وهي كالآتي: 
a a عدد أفراد الجيل الابتدائي : عدد أفراد الجيل يساوي كروموسومين وهما النقطتان المحيطيتان بالنقطـة

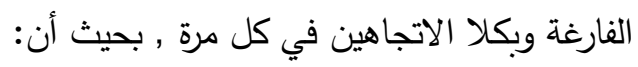

• أفراد الجيل الابتدائي الأفقي هـا الكروموسومان اللذان يمثلان النقطتين المحيطيتين بالنقطـة الفارغـة

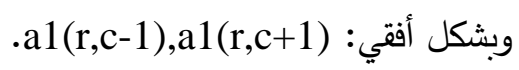

• أفراد الجيل الابتدائي العمودي هـا الكروموسومان اللذان يمثلان النقطتين المحيطيتين بالنقطة الفارغة

$$
\text { وبشكل عمودي: a }
$$

b. طول الكروموسوم : تم اختيار كروموسوم يتألف من 8 جينات لان الصور المستخدمة رمادية وبإمكانها

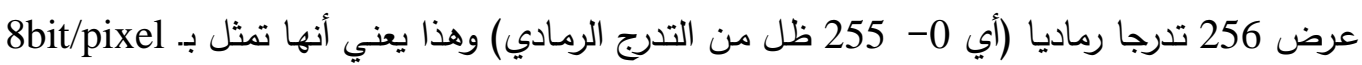

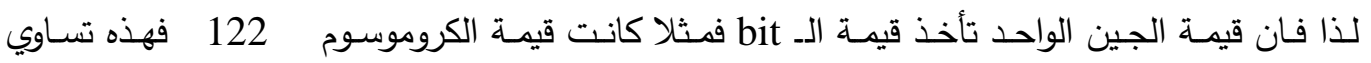
01111010 أي 8 جينات وان قيمة كل bit هي قيمة الجين في هذا الكروموسوم. c ت تحديد نوع الانتقاء وتطبيق التداخل والطفرة. وفيما يلي مثال مبسط لتوضيح الجيل الابتدائي. لتكن لاينا المصفوفة التالية التي تمثل a2:

$\begin{array}{lllll}128 & 0 & 133 & 0 & 127 \\ 0 & 0 & 0 & 0 & 0 \\ 127 & 0 & 80 & 0 & 81 \\ 0 & 0 & 0 & 0 & 0 \\ 111 & 0 & 24 & 0 & 60\end{array}$

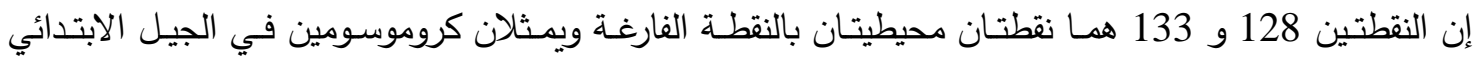
الأفقي ، فبعد إيجاد النقطة الفارغة بينهم 135 حسب خطوات الخوارزمية المقترحة (بينما في الخوارزمية التقليدية سيتم إيجاد المعدل للنقطتين المحيطيتين وهو 130 لتعويضها محل النقطة الفارغة) سوف يتت إيجاد قيمة النقطة الفارغة التالية للجيل الابتدائي الأفقي التالي (133 و 127) وهكذا نستمر إلى أن يتم إيجاد جميع النقاط الفارغة

$\begin{array}{lllll}128 & 135 & 133 & \mathbf{1 3 8} & 127\end{array}$

$\begin{array}{lllll}0 & 0 & 0 & 0 & 0\end{array}$

$\begin{array}{lllll}127 & \mathbf{1 0 0} & 80 & \mathbf{8 3} & 81\end{array}$

$\begin{array}{lllll}0 & 0 & 0 & 0 & 0\end{array}$

$\begin{array}{lllll}111 & 73 & 24 & 47 & 60\end{array}$

يليها يتم تحديد كروموسومات الجيل العمودي الابتدائي ولتكن 128 و 127 المحيطيتين بالنقطة الفارغة والتي بعد إيجاد قيمتها ولتكن 125 حسب خطوات الخوارزمية المقترحة (بينما في الخوارزمية التقليدية سيتم إيجاد المعدل للنقطتين المحيطيتين وهو 127 لتعويضها محل النقطة الفارغة) سيتم تحديد عناصر الجيل العمودي التالي (127 و 111) لإيجاد قيمة النقطة الفارغة بينهم وهكذا تصبح a2 بالصيغة التالية:

\begin{tabular}{lllll}
128 & \multicolumn{1}{c}{135} & \multicolumn{1}{c}{133} & \multicolumn{1}{c}{138} & \multicolumn{1}{c}{127} \\
$\mathbf{1 2 5}$ & $\mathbf{1 1 8}$ & $\mathbf{9 0}$ & $\mathbf{1 0 6}$ & $\mathbf{1 1 4}$ \\
127 & 100 & 80 & 83 & 81 \\
$\mathbf{1 1 7}$ & $\mathbf{8 8}$ & $\mathbf{6 1}$ & $\mathbf{6 2}$ & $\mathbf{7 8}$ \\
111 & 73 & 24 & 47 & 60
\end{tabular}


في هذه الخطوة تم حساب دالة الهدف بالاعتمـاد على المعدل لكل من النقطتين المحيطيتين بالنقطة الفارغة وبكلا الاتجاهين في كل مرة وكالاتي: حساب دالة الهدف أفقيا حسب المعادلة التالية:

$\mathrm{Fr}=(\mathrm{a} 1(\mathrm{r}, \mathrm{c}-1)+\mathrm{a} 1(\mathrm{r}, \mathrm{c}+1)) / 2$

حساب دالة الهدف عموديا حسب المعادلة التالية:

$\mathrm{Fc}=(\mathrm{a} 1(\mathrm{r}-1, \mathrm{c})+\mathrm{a} 1(\mathrm{r}+1, \mathrm{c})) / 2$

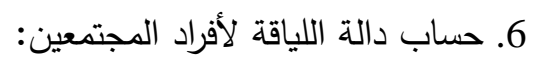

أولا: حساب er أفقيا:

$\operatorname{er}=\operatorname{abs}($ Fr-x $)$

$\mathrm{ec}=\mathrm{abs}(\mathrm{Fc}-\mathrm{y})$

$$
\begin{aligned}
& \text { حيث x احدى النقاط المجتمع الأفقي. } \\
& \text { ثانيا: حساب ec عموديا: }
\end{aligned}
$$

$$
\text { حيث y إحدى النقاط المجتمع العمودي. }
$$

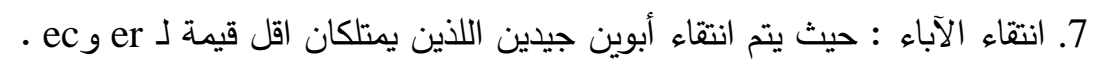

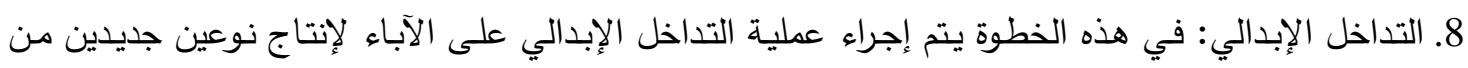

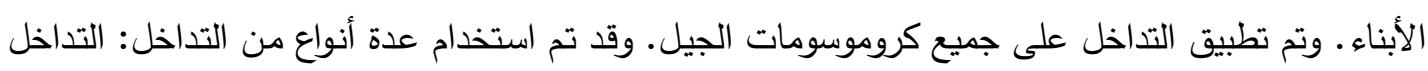

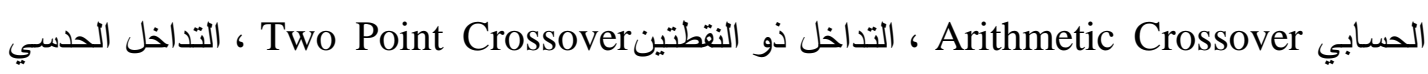
Heuristic Crossover

9. الطفـرة : يـتم تطبيـق الطفرة على الجيـل الجديــــ وتـم اسـتخدام عـدة أنـواع مـن الطفـرات : طفـرة تغييـر

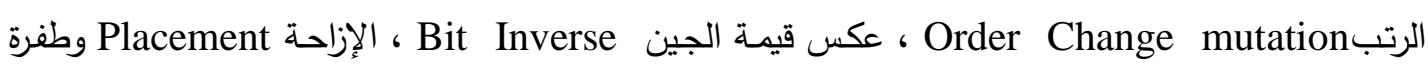
الإضافة والطرح Add or Sub Mutation. ومقدار احتمالية أداء الطفرة المستخدمة في البحث هي 0.5 أي 0.5

$$
\text { على كروموسوم واحد في الجيل المتكون من كروموسومين. }
$$

في البدء تكون مقاطع الجيل الأفقي هما النقطتان المحيطيتان بالنقطة الفارغة (a1(r,c-1),a1(r,c+1)

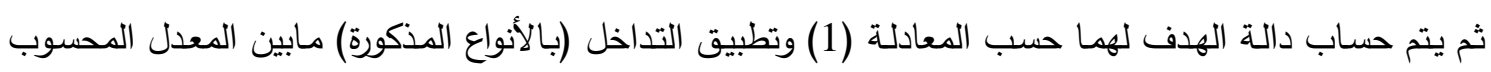
بالمعادلة (1) والنقطة المحيطية الأولى لينتج نقطتين والمعدل مع النقطة المحيطية الثانية لينتج نقطتين أيضا. يليها نحسب اللياقة للنقاط الأربعة حسب المعادلة (3) لكي ننتقي اقرب نقطتين إلى المعدل (النقطتين الأقل لياقة) من

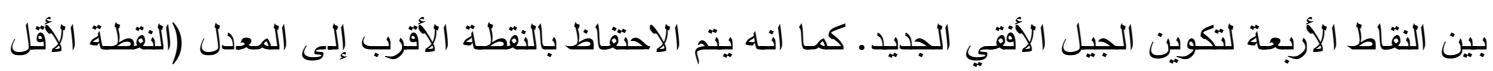

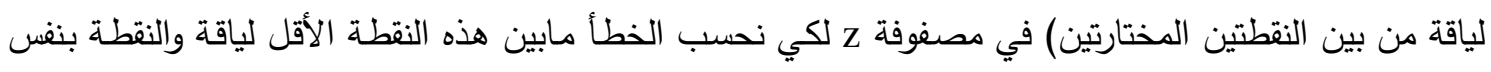

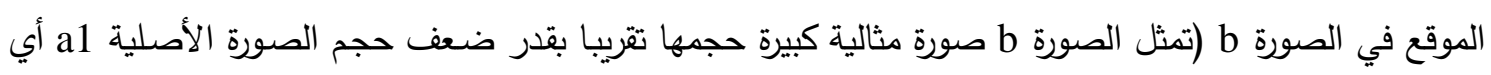

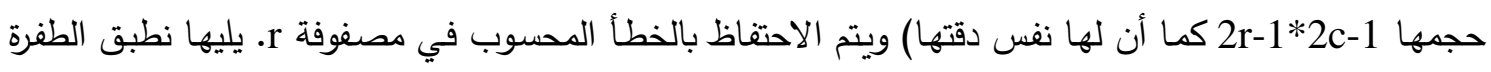
على إحدى النقطتين المختارتين( أي نسبة أداء الطفرة 0.5). ثم نكرر العملية السابقة ويتم التوقف عند الوصول إلى 20 جيل (اعتمادا على نتائج البحث) • يليها يتم اختيار النقطة الأقل خطا محسوبا في مصفوفة r من من بين

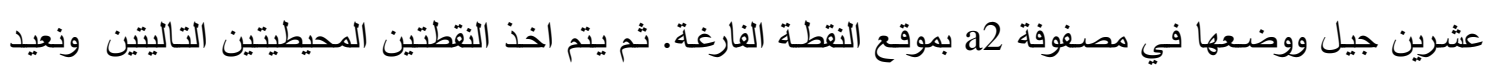
عليهم نفس العملية أعلاه لينتج نقطة بأقل خطا ووضعها أيضـا في مصفوفة a2 وهكذا إلى أن يتم إيجاد جميع النقاط الفارغة في الاتجاه الأفقي بالمصفوفة a2. وبالطريقة أعلاه نفسها يتم تكوين مقاطع الجيل العمودي وإيجاد 
جميع النقاط الفارغة فيه. وبعد الانتهاء من تكوين الصورة الموسعة a2 سوف يتم حساب RMSE و PSNR لها

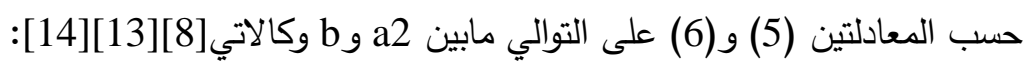
$R M S E=\sqrt{\frac{1}{M * N} \sum_{r=0}^{M-1} \sum_{c=0}^{N-1}\left[\operatorname{Im}_{\text {new }}(r, c)-\operatorname{Im}_{\text {old }}(r, c)\right]^{2}}$

$$
\begin{aligned}
& \text { حيث أن : } \\
& \text { : تمثل أبعاد الصورة : M,N }
\end{aligned}
$$

b تمثل الصورة القديمة (الصورة الأصلية الكبيرة والمثالية Im old $(r, c)$

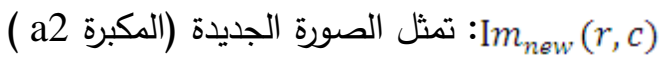

$P S N R=10 \log 10(L-1)^{\wedge} 2 / \frac{1}{M * N} \sum_{r=0}^{M-1} \sum_{c=0}^{N-1}\left[\operatorname{Im}_{n e w}(r, c)-\operatorname{Im}_{\text {old }}(r, c)\right]^{2}$

حيث أن L أكبر تدرج رمادي في الصورة المعالجة. بعد إيجاد RMSE و RSNR للصورة المكبرة الناتجة a2 سيتم إعادة تتفيذ خطوات الخوارزمية من جديد والحصول على صورة مكبرة أخرى a2 ليتم إيجاد RMSE و RSNR لها وهكذا نستمر إلى 20 جيل ، ثم يتم الاحتفاظ وعرض أفضل نتيجة من بين 20 جيلا (عرض الصورة التي تمتلك اقل RMSE واكبر PSNR من بين

ويوضح الثكل(1) المخطط الانسيابي للخوارزمية المصممة.

\section{6. - اختبار الخوارزمية المقترحة}

يجسد اختبار الخوارزمية الهدف الثاني للبحث ، ويعد تحليل النتائج في الوقت نفسه أداة لاختبار فرضية

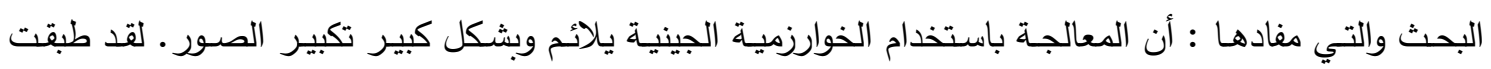
الخوارزمية المقترحة على صور رمادية مختلفة. ولزيادة التوسع في الحصول على نتائج أدق تم تطبيق عدة أنواع

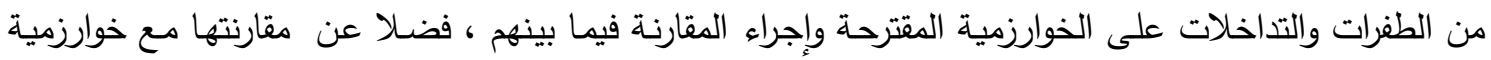
المعدل الخطي التقليدية. واعتمدت جميع المقارنات على مقياسين RMSE و PSNR. وان وان الجدول (1) يمثل أنواع التداخلات والطفرات المستخدمة في الخوارزمية المقترحة إذ تم تثبيت الطفرة وتطبيقها على كل أنواع التداخلات

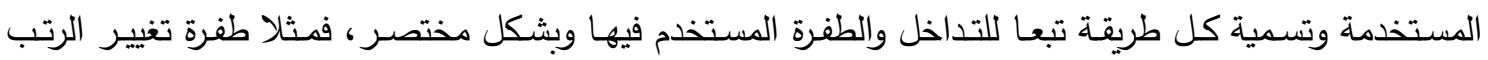

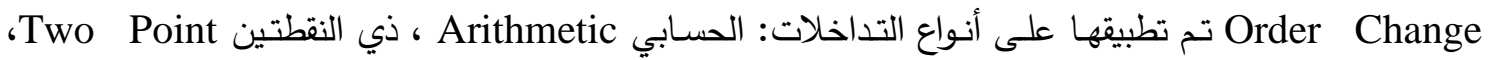
الحسسي Heuristic وذي النقطة الواحدة Single Point. وان الطريقة الأولى

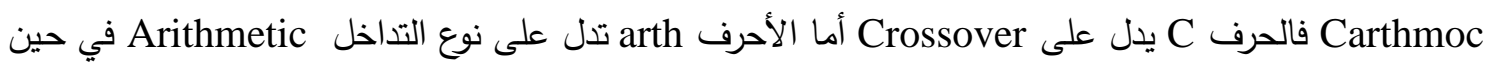

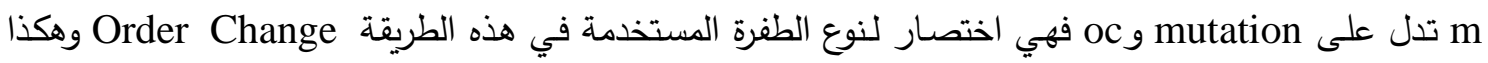
بالنسبة لبقية الطرائق (والتي أسماؤها المختصرة تدل على نوع التداخل والطفرة المستخدمة في تلك الطريقة). 


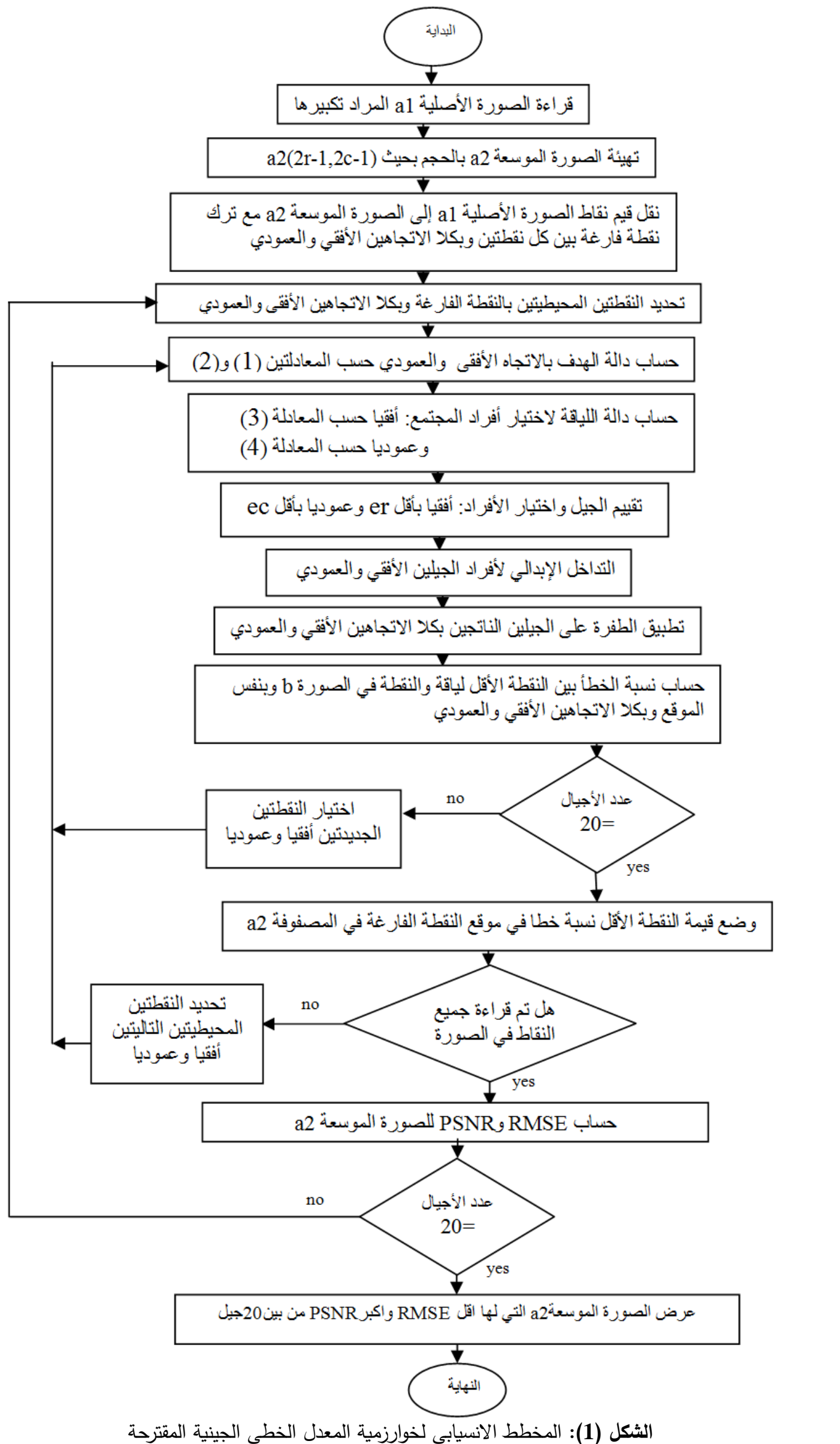


الجدول (1): أنواع التداخل والطفرات المستخدمة في الخوارزمية المقترحة

\begin{tabular}{|l|l|l|l|}
\hline $\begin{array}{c}\text { No. of } \\
\text { Method }\end{array}$ & \multicolumn{1}{|c|}{ Method } & \multicolumn{1}{c|}{ Crossover } & \multicolumn{1}{c|}{ Mutation } \\
\hline Method1 & Carthmoc & Arithmetic Crossover & Order Change Mutation \\
\hline Method2 & Ctwmoc & Two Point Crossover & Order Change Mutation \\
\hline Method3 & Churnoc & Heuristic Crossover & Order Change Mutation \\
\hline Method4 & Cspmoc & Single Point Crossover & Order Change Mutation \\
\hline Method5 & Cathmbi & Arithmetic Crossover & Bit Inverse Mutation \\
\hline Method6 & Ctwpmbi & Two Point Crossover & Bit Inverse Mutation \\
\hline Method7 & Churmbi & Heuristic Crossover & Bit Inverse Mutation \\
\hline Method8 & Cspmbi & Single Point Crossover & Bit Inverse Mutation \\
\hline Method9 & Cathmpl & Arithmetic Crossover & Placement Mutation \\
\hline Method10 & Ctwpmpl & Two Point Crossover & Placement Mutation \\
\hline Method11 & Churmpl & Heuristic Crossover & Placement Mutation \\
\hline Method12 & Cspmpl & Single Point Crossover & Placement Mutation \\
\hline Method13 & Cathmadsu & Arithmetic Crossover & Add or Sub Mutation \\
\hline Method14 & Ctwpmadsu & Two Point Crossover & Add or Sub Mutation \\
\hline Method15 & Churmadsu & Heuristic Crossover & Add or Sub Mutation \\
\hline Method16 & Cspmadsu & Single Point Crossover & Add or Sub Mutation \\
\hline
\end{tabular}

ولتحديد الـ parameters التي تحتاجها كل طريقة من التداخل والمستخدمة في البرنامج فهي كالاتي:

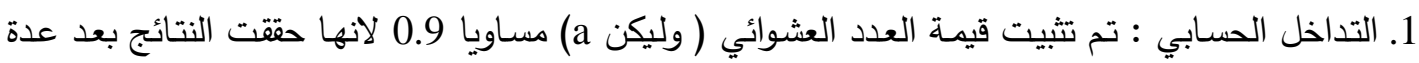
محاولات من اختيار قيم عشوائية اخرى محصورة بين 0 و 1 وكذلك لغرض إجراء المقارنات بين هذه الطرائق المتتوعة من التداخلات والطغرات.

2. التداخل ذو النقطتين : تم اختيار نقطتين (مسقعين) الذين عندهما يتم التبديل بين قيم الـ bits ( أي الجينات الواقعة بين الموقعين المحددين) ضمن مقطعي الاباء.

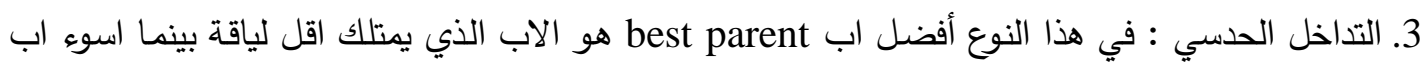

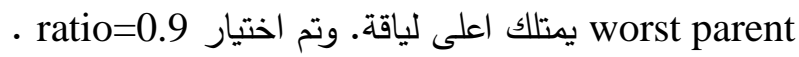
4. التداخل ذو النقطة الواحدة : تم تحديد نقطة (موقع) الذي عنده يتم التبديل بين قيم الـ bits (أي الجينات المتقابلة) ضمن مقطعي الاباء. اما بالنسبة لا parameters التي تم اعتمادها في الطفرات فهي كالاتي: 1. طفرة تغيير الرتب :تم اختيار موقعين في الكروموسوم الواحد ثم مبادلة قيم الـ bits ببعضهما.

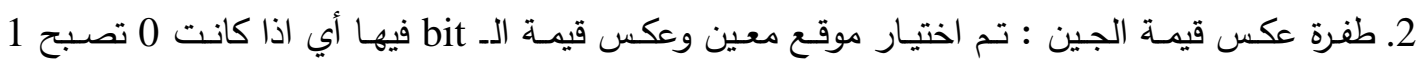
وبالعكس.

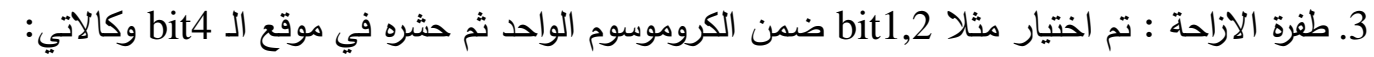

\begin{tabular}{|c|c|c|c|}
\hline 01010011 & قيم الـ bits مثلا & $876543(\mathbf{2 1})$ & مواقع الـ bits في الكروموسوم \\
\hline 01011100 & بعد تطبيق الطفرة عذد موقع bit4 تصبح & $8765(\mathbf{2 1}) 43$ & بعد تطبيق الطفرة عند موقع bit4 \\
\hline
\end{tabular}

4. طفرة الاضـافة والطرح : تم اضـافة وطرح عدد صغير (اضـافة 0.5 وطرح 0.6) لقيم two bit ضمن

$$
\text { الكروموسوم الواحد وتقريبها إلى الصغر والواحد. }
$$


لقد تم تطبيق خوارزمية المعدل الخطي الجينية المقترحة وخوارزمية المعدل الخطي التقليدية على صورة Flower.png و Cameraman.tif

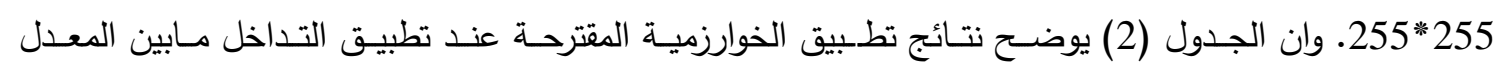

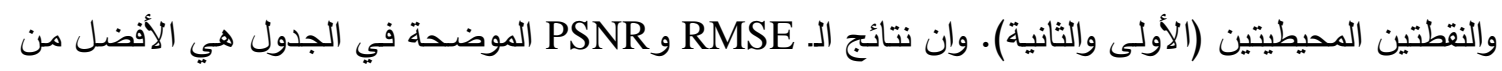
بين 20 جيلا ، والعمود No. of generation يمثل رقم الجيل الذي عنده تم الحصول على هذه النتائج . الجدول (2): نتائج تطبيق الخوارزمية الجينية المقترحة عند تطبيق التداخل مابين المعدل وكلتا النقطتين المحيطيتين.

\begin{tabular}{|l|c|l|c|c|c|c|}
\hline \multicolumn{4}{|c}{ Cameraman.tif } & \multicolumn{3}{c|}{ Flower.png } \\
\hline Method & $\begin{array}{c}\text { PSNR } \\
(\mathbf{d b})\end{array}$ & $\mathbf{R M S E}$ & $\begin{array}{c}\text { No. of } \\
\text { generation }\end{array}$ & $\begin{array}{c}\text { PSNR } \\
(\mathbf{d b})\end{array}$ & $\mathbf{R M S E}$ & $\begin{array}{c}\text { No. of } \\
\text { generation }\end{array}$ \\
\hline Method1:Carthmoc & $\mathbf{2 9 . 2 9 5 5}$ & $\mathbf{8 . 7 4 5 2}$ & $\mathbf{4}$ & $\mathbf{2 9 . 6 6 2 8}$ & $\mathbf{8 . 5 2 7 7}$ & $\mathbf{3}$ \\
\hline Method2:Ctwmoc & 29.1847 & 8.8574 & 5 & 29.5423 & 8.6789 & 4 \\
\hline Method3:Churnoc & 27.7011 & 10.5071 & 16 & 29.5911 & 8.5965 & 13 \\
\hline Method4:Cspmoc & 29.2091 & 8.8326 & 1 & 29.5777 & 8.6170 & 2 \\
\hline Method5:Cathmbi & 29.1742 & 8.8571 & 6 & 29.6422 & 8.5422 & 4 \\
\hline Method6:Ctwpmbi & 29.1653 & 8.7732 & 2 & 29.5561 & 8.6591 & 6 \\
\hline Method7:Churmbi & 27.7921 & 10.3931 & 3 & 29.2324 & 8.7200 & 6 \\
\hline Method8:Cspmbi & 29.2291 & 8.6270 & 1 & 29.5666 & 8.6270 & 2 \\
\hline Method9:Cathmpl & 29.1158 & 8.9279 & 1 & 29.6216 & 8.5513 & 3 \\
\hline Method10:Ctwpmpl & 29.1340 & 8.9093 & 1 & 29.5443 & 8.6732 & 1 \\
\hline Method11:Churmpl & 27.7958 & 10.3932 & 14 & 29.2413 & 8.7043 & 6 \\
\hline Method12:Cspmpl & 29.2091 & 8.8326 & 1 & 29.6167 & 8.5744 & 4 \\
\hline Method13:Cathmadsu & 29.2381 & 8.9379 & 4 & 29.5352 & 8.6950 & 1 \\
\hline Method14:Ctwpmadsu & 29.1489 & 8.8321 & 1 & 29.5565 & 8.6511 & 2 \\
\hline Method15:Churmadsu & 27.8701 & 10.3091 & 2 & 29.1184 & 8.9654 & 6 \\
\hline Method16:Cspmadsu & 29.1891 & 8.8779 & 6 & 29.5657 & 8.6278 & 2 \\
\hline
\end{tabular}

وعند تطبيق التداخل مـابين المعدل واحدى النقاط المحيطيـة (النقطسة المحيطية الأولى) لينتج نقطتين واعتبارهم عناصر الجيل (بدلا من اربع نقاط واختيار نقطتين تمتلكان اقل لياقة) ، ظهرت النتائج المبينة في الجدول (3). وتوضح الاشكال (2،2) نتائج تطبيق الخوارزمية المقترحة.

الجدول (3): نتائج تطبيق الخوارزمية الجينية المقترحة عند تطبيق التداخل مابين المعدل والنقطة المحيطية الاولى الى

\begin{tabular}{|l|c|c|c|c|c|c|}
\hline \multicolumn{4}{|c|}{ Cameraman.tif } & \multicolumn{3}{c|}{ Flower.png } \\
\hline Method & $\begin{array}{c}\text { PSNR } \\
(\mathbf{d b})\end{array}$ & $\mathbf{R M S E}$ & $\begin{array}{c}\text { No. of } \\
\text { generation }\end{array}$ & $\begin{array}{c}\text { PSNR } \\
(\mathbf{d b})\end{array}$ & RMSE & $\begin{array}{c}\text { No. of } \\
\text { generation }\end{array}$ \\
\hline Method1:Carthmoc & 31.6099 & 6.6996 & 4 & 31.1203 & 7.0800 & 4 \\
\hline Method2:Ctwmoc & 31.2840 & 6.9557 & 6 & 30.6150 & 7.5126 & 4 \\
\hline Method3:Churnoc & 29.2719 & 8.7629 & 1 & 29.6518 & 8.4031 & 2 \\
\hline Method4:Cspmoc & $\mathbf{3 1 . 6 4 7 0}$ & $\mathbf{6 . 6 5 4 1}$ & $\mathbf{4}$ & $\mathbf{3 1 . 1 5 4 1}$ & $\mathbf{7 . 0 6 0 5}$ & $\mathbf{4}$ \\
\hline Method5:Cathmbi & 31.5874 & 6.7169 & 4 & 31.1325 & 7.0781 & 4 \\
\hline Method6:Ctwpmbi & 31.3215 & 6.9257 & 5 & 30.3494 & 7.7459 & 4 \\
\hline Method7:Churmbi & 29.2671 & 8.7609 & 1 & 29.6213 & 8.5031 & 3 \\
\hline Method8:Cspmbi & 31.5933 & 6.7123 & 4 & 31.1362 & 7.0751 & 4 \\
\hline Method9:Cathmpl & 30.6042 & 7.5219 & 8 & 29.6318 & 8.4130 & 1 \\
\hline Method10:Ctwpmpl & 29.2714 & 8.7599 & 1 & 29.6513 & 8.3130 & 2 \\
\hline Method11:Churmpl & 29.2699 & 8.7599 & 1 & 30.5555 & 7.9013 & 2 \\
\hline Method12:Cspmpl & 31.0116 & 7.1773 & 6 & 29.6518 & 8.4013 & 3 \\
\hline Method13:Cathmadsu & 30.6765 & 7.4596 & 5 & 30.0374 & 8.0291 & 5 \\
\hline
\end{tabular}




\begin{tabular}{|l|l|l|l|l|l|l|}
\hline Method14:Ctwpmadsu & 31.0592 & 7.1381 & 5 & 30.4198 & 7.6833 & 4 \\
\hline Method15:Churmadsu & 29.2711 & 8.7499 & 1 & 29.6318 & 8.4030 & 1 \\
\hline Method16:Cspmadsu & 31.3483 & 6.9044 & 4 & 29.5657 & 8.6278 & 2 \\
\hline
\end{tabular}

اما الجدول (4) يبين نتائج خوارزمية المعدل الخطي التقليدية.

الجدول (4): نتائج تطبيق خوارزمية المعدل الخطي التقليدية

\begin{tabular}{|c|c|c|}
\hline PSNR & RMSE & image \\
\hline 29.2642 & 8.7767 & Cameraman.tif \\
\hline 29.6370 & 8.4080 & Flower.png \\
\hline
\end{tabular}

وبامكان الخوارزمية المقترحة ان تكبر الصورة اكثر وحسب رغبة المستخدم وذلك باعادة تتفيذها على أفضل صورة

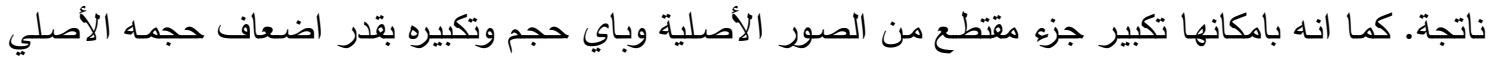

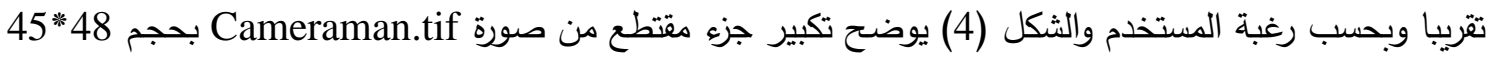

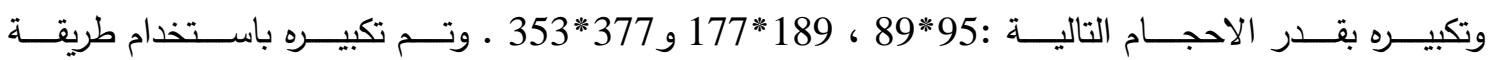
. بالتداخل الثاني (تداخل المعدل مع احدى النقاط المحيطية) : Method4(Cspmoc)

7. الاستنتاجات والبحوث المستقبلية (Conclusions \& Future Research)

استتاداً إلى ما تم عرضسه في الجانب النظري والجانب التطبيقي وكما في الجداول (2،3،2) والاشكال(4،3، (4، يمكن تأثير أهم الاستتاجات :

1. عند المقارنـة بين الجدولين (2) مـع (3) تبين ان التداخل بالطريقـة الثانية(تداخل المعدل مـع احدى النقاط

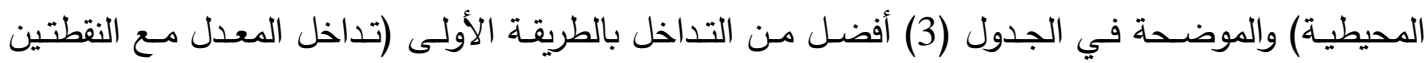

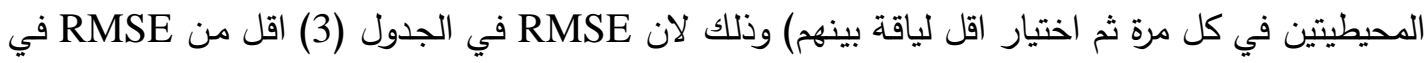

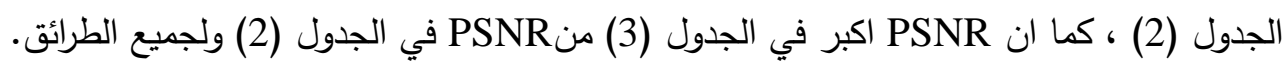
2. عند تطبيق الخوارزمية الجينية المقترحة على عدة أنواع من التداخل والطفرات تبين ان هنالك فروقات طفيفة بينهم بشكل عام.

3. تبين من الجدول (2) ان أفضل طريقة هي Method1(Carthmoc) (التداخل الحسابي وطفرة تغيير

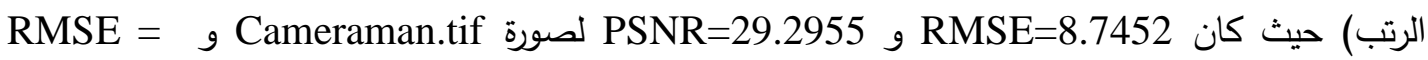

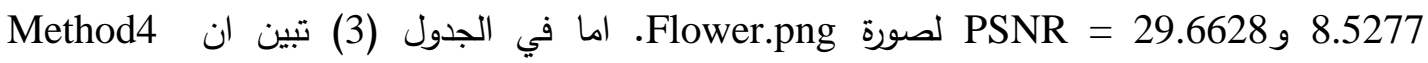
و (تداخل ذا النقطة الواحدة وطفرة تغيير الرتب) هي الأفضل حيث كان 2 (Cspmoc)

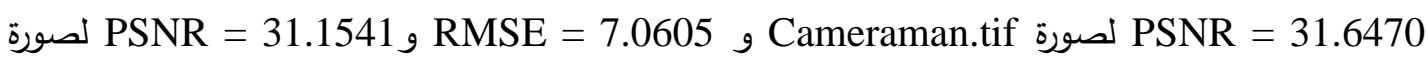
Method4 وعlower.png (تداخل ذا النقطة الواحدة وطفرة تغيير الرتب) الأكثر ملائمة من الأنواع الاخرى. وعند المقارنة

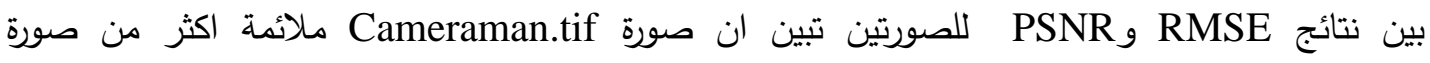

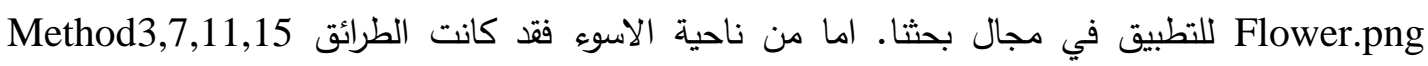
(تداخلا حدسيا مع جميع أنواع الطفرات) الاسوء مقارنة بباقي الطرائق في كلا الجدولين وبشكل عام .

4. مما سبق نستتج إنٍ الخوارزمية الجينية المقترحة بكافة طرائقها وبالاخص (Method4(Cspmoc (تداخل ذا

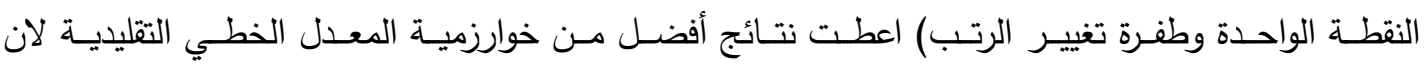




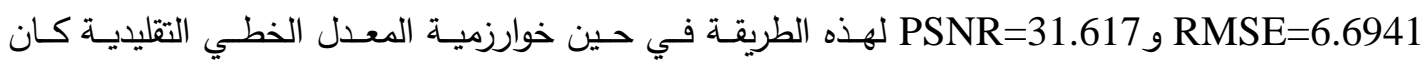

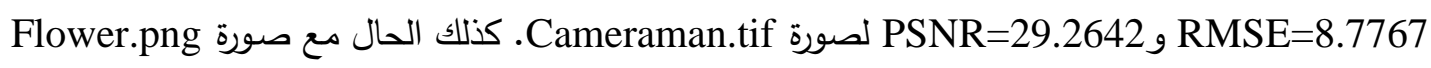
التي ظهرت نتائجها في الخوارزمية الجينية أفضل من التقليدية بالنسبة للمقياسين.

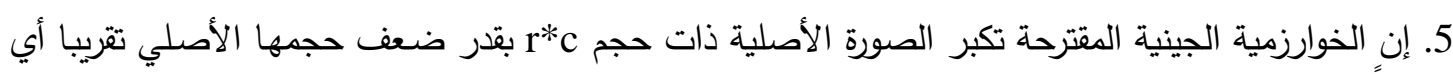

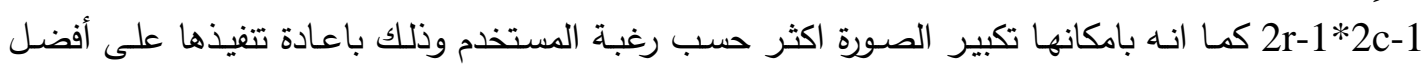
صورة ناتجة منها. ايضا بامكانها تكبير أي جزء مقتطع من الصورة الأصلية وحسب رغبة رغبة المستخدم. اما البحوث المستقبلية فهي: تهجين طرائق اخرى لتكبير الصور مع الخوارزمية الجينية. تكبير الصور الملونة. تحسين الصورة بعد تكبيرها. استخدام طرائق ذكائية اخرى في تكبير الصور . 


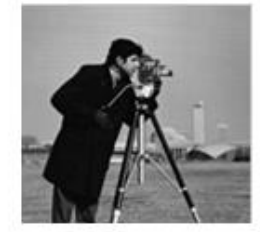

Small original image (a1)

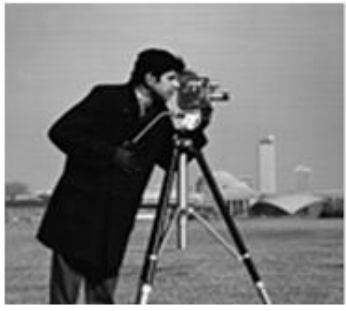

Big original image (b)

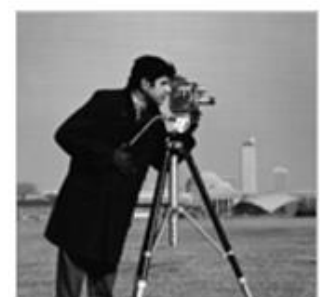

Method1: Carthmoc

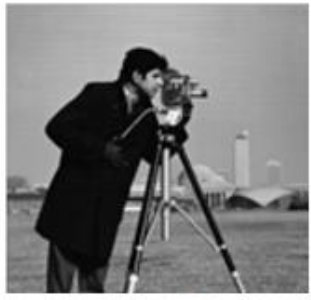

Method5: Cathmbi

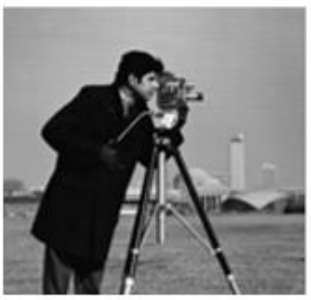

Method9: Cathmp1

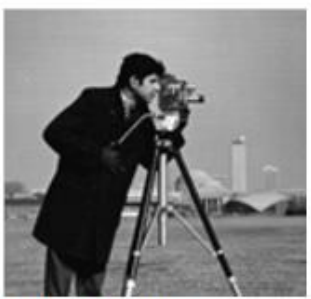

Method 13: Cathmadsu

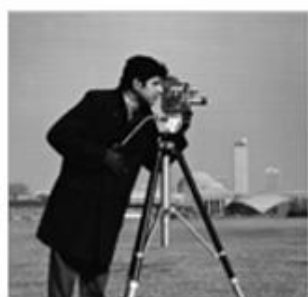

Method2: Ctwmoc

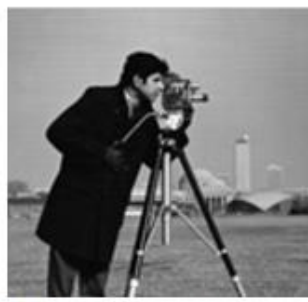

Method6: Ctwpmbi

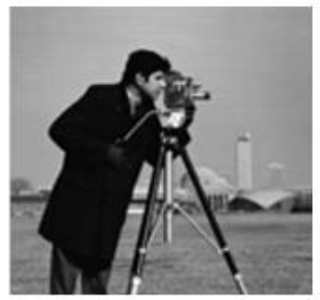

Method10: Ctwpmp1

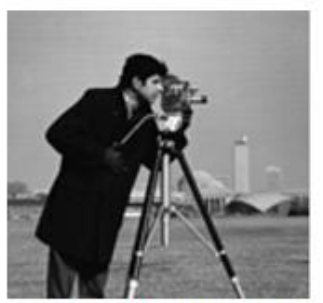

Method14:Ctwpmadsu

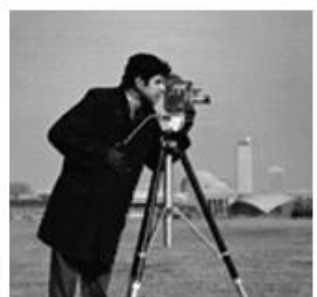

Method3: Churnoc

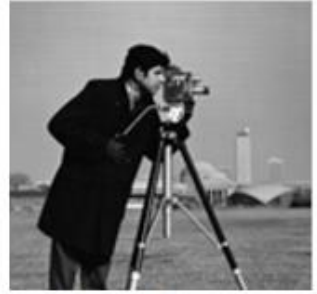

Method7: Churmbi

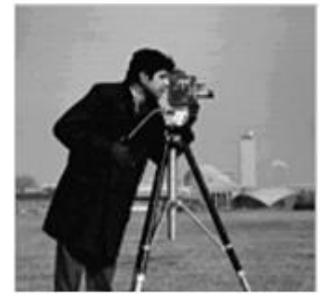

Method 11: Churmpl

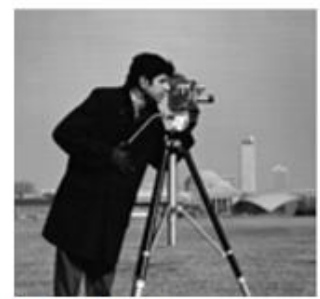

Method15: Churmadsu

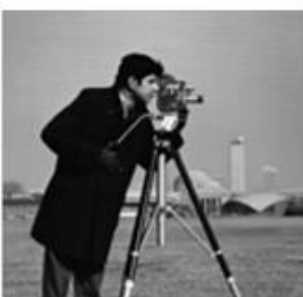

Method4: Cspmoc

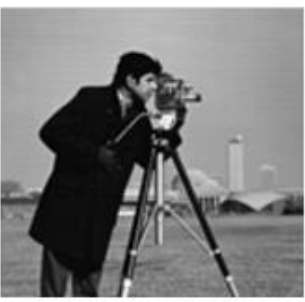

Method8: Cspmbi

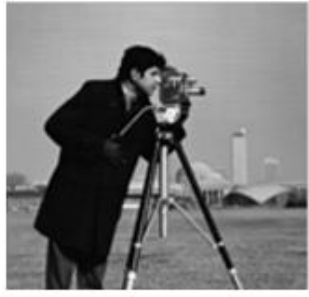

Method12: Cspmpl

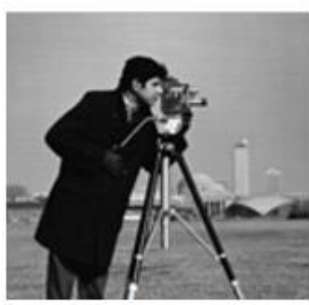

Method 16:Cspmadsu

Cameraman.tif الثكل (2): نتائج تطبيق الخوارزمية الجينية المقترحة على صورةC 


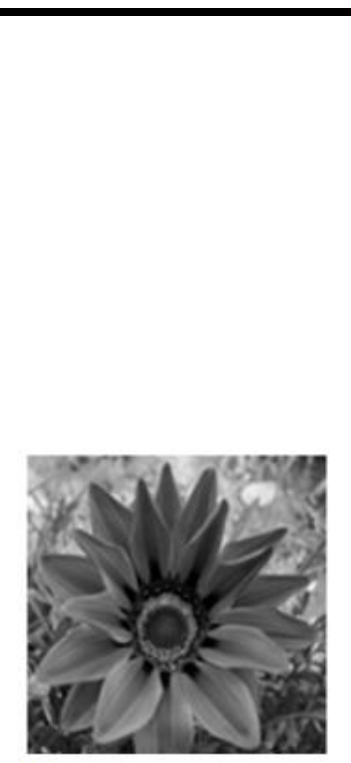

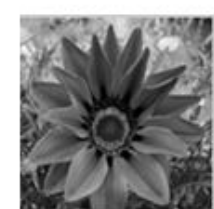

Small original image (a1)

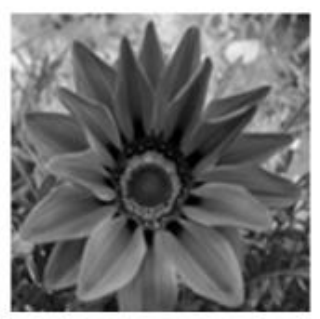

Method2: Ctwmoc

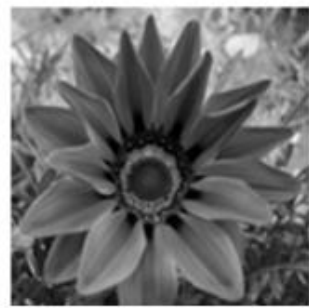

Method6: Ctwpmbi

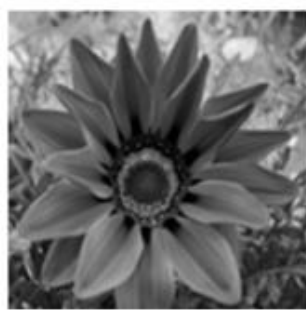

Method10: Ctwpmpl

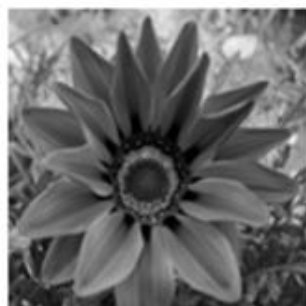

Method14:Ctwpmadsu

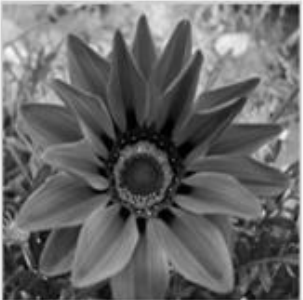

Big original image (b)

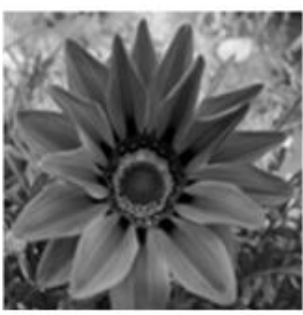

Method3: Churnoc

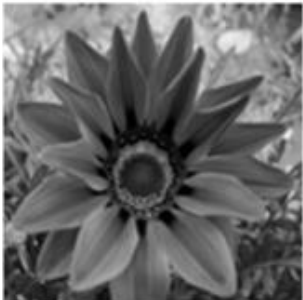

Method7: Churmbi

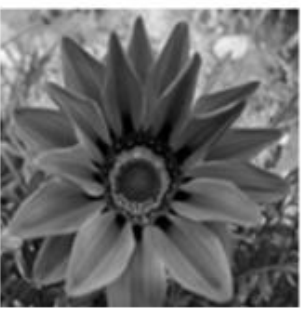

Method 11: Churmp1

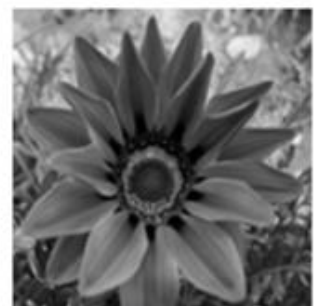

Method15: Churmadsu

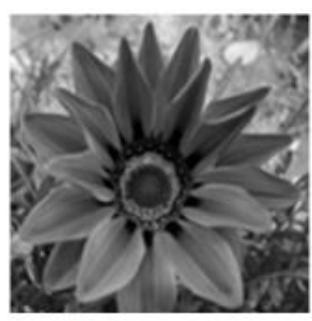

Method4: Cspmoc

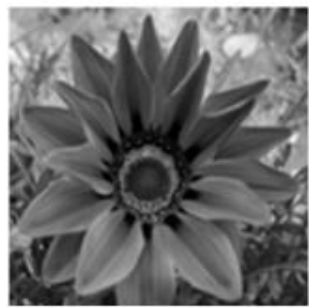

Method8: Cspmbi

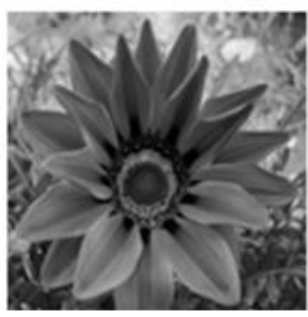

Method12: Cspmp1

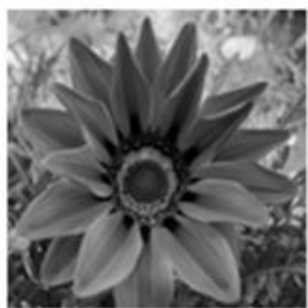

Method 16:Cspmadsu

Flower.png الثكل (3): نتائج تطبيق الخوارزمية الجينية المقترحة على صورة 


\section{A}

original image
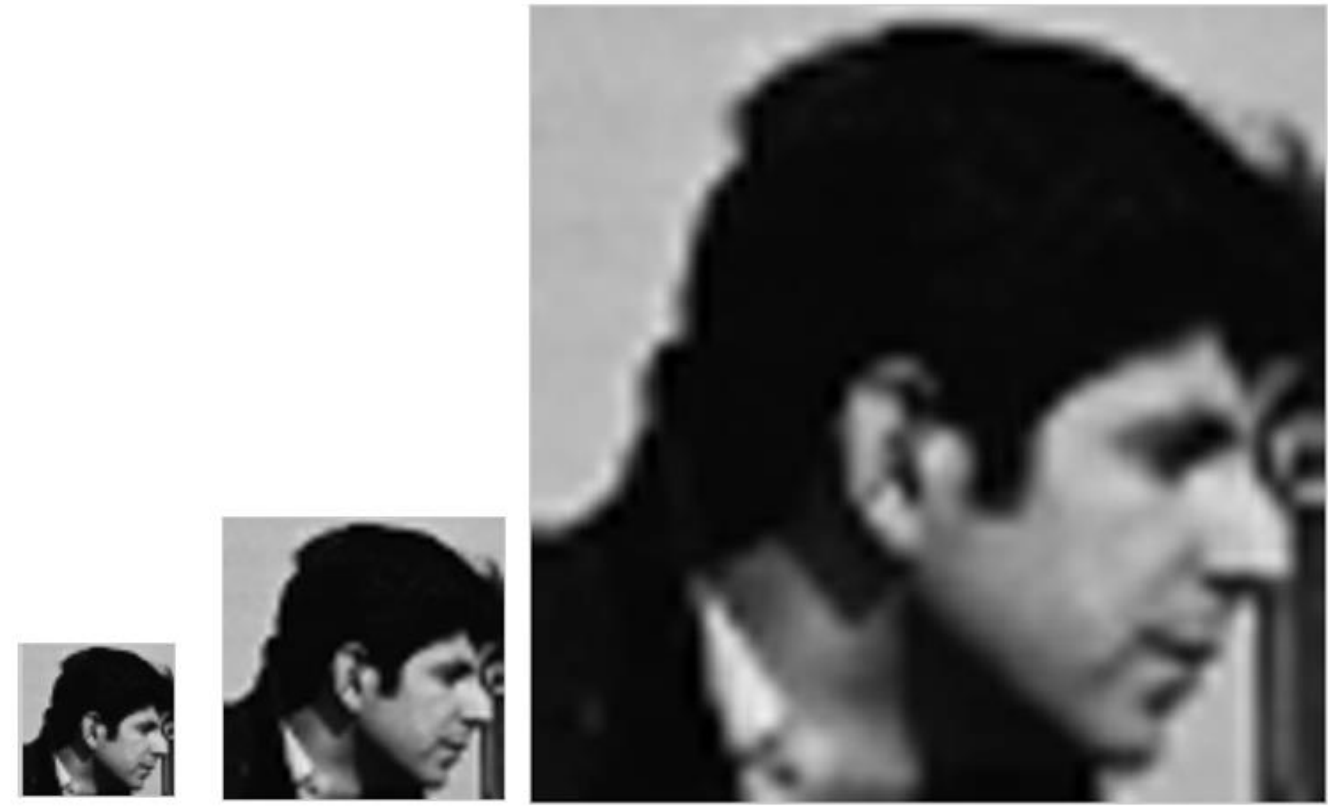

الثكل(4): نتائج تطبيق الخوارزمية الجينية المقترحة بطريقة Method4: Cspmoc على جزء مقتطع من صورة Cameraman.tif 


\section{المصادر}

[1] Al-Mamun ,H.A., Jahangir ,N., Islam M.S and Islam ,M.A.,(2009),"Eye Detection in Facial Image by Genetic Algorithm Driven Deformable Template Matching" IJCSNS International Journal of Computer Science and Network Security, Vol.9 No.8,pp287-294.

[2] Almira, J. M and Romero,,A. E., (2011) ,"Image Zooming based on sampling theorems" MAT2,ISSN: 1887-1097, Publicaci_o electr_onica de divulgaci_o del Departament de Matem_atiques de la Universitat Aut_onoma de Barcelona sampling theorems, Vol 2011, pp. 1- 22 .

[3] Battiato,S,Gallo,G. and Stanco,F. ,(2002)" A locally adaptive zooming algorithm for digital images", Published by Elsevier Science B.V., Image and Vision Computing20,pp. 805-812.

[4] Furtado,J.,J. , Cai1,Z. and Xiaobo,L.,(2010)," Digital Image Processing: Supervised Classification Using Genetic Algorithm In Matlab Toolbox", ISSN:1553-9873, Report and Opinion2(6)pp.53-61.

[5] Gao, R., Ping Song, Jin, And Cheng Tai, X., ,(2009),"Image Zooming Algorithm Based On Partial Differential Equations Technique", International Journal Of Numerical Analysis And Modeling. Institute for Scientific Computing and Information, Vol. 6, No. 2, pp. 284-292.

[6] Getreuer ,P.,(2009),"Image zooming with contour stencils",University of California Los Angeles, Mathematics Department, U.S.A. Computational Imaging VII, SPIE-IS\&T/ Vol. 7246, 72460,pp.1-12.

[7] Gonzales, R. C. and Woods, R E., ( 2002), "Digital Image processing", Second Edition, Prentice Hall, New Jersey 07458.

[8] Horé,A and Ziou,D., (2010)," Image quality metrics: PSNR vs. SSIM ", International Conference on Pattern Recognition ,IEEE Computer Society,pp. 2358-2369.

[9] Karkavitsas,G. and and Rangoussi,M.,(2005),"Object localization in medical images using genetic algorithms",World Academy of Science, Engineering and Technology Vol.2,pp.6-9.

[10] Kussener,F.,(2011)," Active contour: a parallel genetic algorithm approach", ICSI: International conference on swarm intelligence,pp.1-9.

[11] Pal ,S.and Hazra, L. N., (2009)," Optically Compensated Zoom Lenses: GA Based Structural Design", ICOP- International Conference on Optics and Photonics, CSIO,Chandigarh, India,pp 1-4.

[12] Raheema,M.N.,(2011)," Advanced Neighborhood Operation Based Image Zooming In", Eng.\&Tech.Journal,Vol.29,No.4,pp.791-804.

[13] Santa-Cruza, D., Ebrahimia, T., Askelöfb J., Larssonb, M.and Christopoulosb C. A., (2000),"JPEG 2000 still image coding versus other standards", In Proc. of the SPIE's 45th annual meeting, Applications of Digital Image Processing XXIII, volume 4115, pages 446-454. 
[14] Sharma,R., Walia,E and Sharma, D.,(2011)," Analysis Of Non-Adaptive And Adaptive Edge Based Lsb Steganography For Colored Images", International Journal of Computing and Business Research (IJCBR) ISSN (Online) : 22296166,Vol. 2, Issue 1.

[15] Srikanth,V., Asati,U., Natarajan,V.,Kumar,P.T, Mullapudi,T and Iyengar, N.Ch.S.N.,(2010)," Bit-Level Encryption of Images using Genetic Algorithm", TECHNIA International Journal of Computing Science and Communication Technologies, Vol. 3, No. 1, (ISSN 0974-3375),pp. 546- 550.

[16] Umbangh,Scott E., 1998,"Computer Vision and Image Processing",Prentice Hall PTR.

[17] Valizadeh, R., Mahmoodabadi, M. J., Ketabchi, S. and Kianpour,M.,(2012)," An Image Zooming Technique Based Moving Least Squares Using Spline and Exponential Weights", Research Article,Academic Editors: S. Gong and J. Kou. 